\title{
Evanescent Effects can Alter Ultraviolet Divergences in Quantum Gravity without Physical Consequences
}

\author{
Zvi Bern, ${ }^{1,2,3}$ Clifford Cheung, ${ }^{2}$ Huan-Hang Chi, ${ }^{4}$ Scott Davies, ${ }^{1}$ Lance Dixon, ${ }^{2,4}$ and Josh Nohle ${ }^{1}$ \\ ${ }^{1}$ Department of Physics and Astronomy, University of California at Los Angeles, Los Angeles, California 90095, USA \\ ${ }^{2}$ Walter Burke Institute for Theoretical Physics, California Institute of Technology, Pasadena, California 91125, USA \\ ${ }^{3}$ Department of Physics, CERN Theory Division, CH-1211 Geneva 23, Switzerland \\ ${ }^{4}$ SLAC National Accelerator Laboratory, Stanford University, Stanford, California 94309, USA
}

(Received 3 August 2015; published 17 November 2015)

\begin{abstract}
Evanescent operators such as the Gauss-Bonnet term have vanishing perturbative matrix elements in exactly $D=4$ dimensions. Similarly, evanescent fields do not propagate in $D=4$; a three-form field is in this class, since it is dual to a cosmological-constant contribution. In this Letter, we show that evanescent operators and fields modify the leading ultraviolet divergence in pure gravity. To analyze the divergence, we compute the two-loop identical-helicity four-graviton amplitude and determine the coefficient of the associated (nonevanescent) $R^{3}$ counterterm studied long ago by Goroff and Sagnotti. We compare two pairs of theories that are dual in $D=4$ : gravity coupled to nothing or to three-form matter, and gravity coupled to zero-form or to two-form matter. Duff and van Nieuwenhuizen showed that, curiously, the one-loop trace anomaly - the coefficient of the Gauss-Bonnet operator - changes under $p$-form duality transformations. We concur and also find that the leading $R^{3}$ divergence changes under duality transformations. Nevertheless, in both cases, the physical renormalized two-loop identical-helicity four-graviton amplitude can be chosen to respect duality. In particular, its renormalization-scale dependence is unaltered.
\end{abstract}

Although theories of quantum gravity have been studied for many decades, basic questions about their ultraviolet (UV) structure persist. One subtlety is the trace anomaly [1] which, at one loop, provides the coefficient of the GaussBonnet (GB) term. The physical significance of this relationship has not been settled, however. In particular, Duff and van Nieuwenhuizen showed that the trace anomaly changes under duality transformations of $p$-form fields, suggesting that theories related through such transformations are quantum-mechanically inequivalent [2]. In response, Siegel argued that this effect is a gauge artifact and, therefore, not physical [3]; Fradkin, Tseytlin, Grisaru et al. have also argued that duality should hold at the quantum level [4]. Furthermore, for $D=4$ external states, one-loop divergences in gravity theories coupled to two-form antisymmetric tensors are unchanged under a duality transformation relating two-forms to zero-form scalars [5]. However, as we shall see, intuition based on one-loop analyses can be deceptive.

As established in the seminal work of 't Hooft and Veltman [6], pure gravity is finite at one loop because the only available on-shell counterterm is the GB term, which integrates to zero in a topologically trivial background. While amplitudes with external matter fields diverge at one loop, amplitudes with only external gravitons remain finite. At two loops, however,

Published by the American Physical Society under the terms of the Creative Commons Attribution 3.0 License. Further distribution of this work must maintain attribution to the author(s) and the published article's title, journal citation, and DOI. pure gravity diverges, as demonstrated explicitly by Goroff and Sagnotti [7] and confirmed by van de Ven [8].

In this Letter, we investigate the UV properties of the two-loop amplitude for the scattering of four identicalhelicity gravitons, including the effect of $p$-form duality transformations. We use dimensional regularization, which forces us to consider the effects of evanescent operators like the GB term. By definition, an evanescent operator is independent of other operators in $D$ dimensions, but either vanishes, or is a total derivative, or becomes a linear combination of other operators in four dimensions. We show that the GB counterterm is required to cancel subdivergences and reproduce the two-loop counterterm coefficient found previously $[7,8]$.

Evanescent operators are well studied in gauge theory (see, e.g., Ref. [9]), where they can modify subleading corrections. In contrast, we find that evanescent effects can alter the leading UV divergence in gravity. (Effects of the GB term have also been studied in renormalizable, but nonunitary, $R^{2}$ gravity [10].) Despite this change in the UV divergence, the physical dependence of the renormalized amplitude on the renormalization scale remains unchanged. This break in the link between the UV divergence and the renormalization-scale dependence is unlike familiar oneloop examples. We arrive at a similar conclusion when comparing the divergences and renormalization-scale dependences in gravity coupled to scalars versus antisymmetric-tensor fields.

Pure gravity is defined by the Einstein-Hilbert (EH) Lagrangian 


$$
\mathcal{L}_{\mathrm{EH}}=-\frac{2}{\kappa^{2}} \sqrt{-g} R
$$

where $\kappa^{2}=32 \pi G_{N}=32 \pi / M_{P}^{2}$ and the metric signature is $(+---)$. We also augment $\mathcal{L}_{\mathrm{EH}}$ by matter Lagrangians for one of the following: $n_{0}$ scalars, $n_{2}$ two-form fields (antisymmetric tensors), or $n_{3}$ three-form fields,

$$
\begin{gathered}
\mathcal{L}_{0}=\frac{1}{2} \sqrt{-g} \sum_{j=1}^{n_{0}} \partial_{\mu} \phi_{j} \partial^{\mu} \phi_{j}, \quad \mathcal{L}_{2}=\frac{1}{6} \sqrt{-g} \sum_{j=1}^{n_{2}} H_{j \mu \nu \rho} H_{j}^{\mu \nu \rho}, \\
\mathcal{L}_{3}=-\frac{1}{8} \sqrt{-g} \sum_{j=1}^{n_{3}} H_{j \mu \nu \rho \sigma} H_{j}^{\mu \nu \rho \sigma} .
\end{gathered}
$$

Here, $\phi_{j}$ is a scalar field and $H_{j \mu \nu \rho}$ and $H_{j \mu \nu \rho \sigma}$ are the field strengths of the two- and three-form antisymmetric-tensor fields $A_{j \mu \nu}$ and $A_{j \mu \nu \rho}$. The index $j$ labels distinct fields. Standard gauge fixing for the two- and three-form actions, as well as for $\mathcal{L}_{\mathrm{EH}}$, leads to a nontrivial ghost structure. We avoid such complications by using the generalized unitarity method [11-13], which directly imposes appropriate $D$-dimensional physical-state projectors on the on-shell states crossing unitarity cuts.

Under a duality transformation, in four dimensions, the two-form field is equivalent to a scalar,

$$
H_{j \mu \nu \rho} \leftrightarrow \frac{i}{\sqrt{2}} \varepsilon_{\mu \nu \rho \alpha} \partial^{\alpha} \phi_{j},
$$

and the three-form field is equivalent to a cosmologicalconstant contribution via

$$
H_{j \mu \nu \rho \sigma} \leftrightarrow \frac{2}{\sqrt{3}} \varepsilon_{\mu \nu \rho \sigma} \frac{\sqrt{\Lambda_{j}}}{\kappa} .
$$

As usual, we expand the graviton field around a flat-space background: $g_{\mu \nu}=\eta_{\mu \nu}+\kappa h_{\mu \nu}$. Similarly, we expand the scalar, two-form field, and three-form field around trivial background values. It is interesting to note that the threeform field has been proposed as a means for neutralizing the cosmological constant [14].

For a theory with $n_{0}$ scalars, $n_{2}$ two-forms and $n_{3}$ threeforms coupled to gravity, the one-loop UV divergence takes the form of the GB term $[1,2,7]$

$$
\begin{aligned}
\mathcal{L}_{\mathrm{GB}}= & \frac{1}{(4 \pi)^{2}} \frac{1}{\epsilon}\left(\frac{53}{90}+\frac{n_{0}}{360}+\frac{91 n_{2}}{360}-\frac{n_{3}}{2}\right) \\
& \times \sqrt{-g}\left(R^{2}-4 R_{\mu \nu}^{2}+R_{\mu \nu \rho \sigma}^{2}\right),
\end{aligned}
$$

which is proportional to the trace anomaly. The calculations of the trace anomaly and of the UV divergence are essentially the same, except that we replace a graviton polarization tensor with a trace over indices. The matrix elements produced by Eq. (5) vanish for four on-shell $D=4$ graviton polarization tensors. This is because the GB combination is evanescent in $D=4$ : It is a total derivative and vanishes when integrated over a topologically trivial space; hence, pure Einstein gravity is finite at one loop [6]. In a topologically nontrivial space, the integral over the GB term gives the Euler characteristic. When matter is added to the theory, the four-graviton amplitude is still UV finite at one loop, although divergences appear in amplitudes with external matter states.

Using the unitarity method, we verified Eq. (5) by considering the one-loop four-graviton amplitude with external states in arbitrary dimensions and internal ones in $D=4-2 \epsilon$ dimensions. On-shell scattering amplitudes are sensitive only to the coefficient of the $R_{\mu \nu \rho \sigma}^{2}$ operator, because the $R^{2}$ and $R_{\mu \nu}^{2}$ operators can be eliminated by field redefinitions at leading order in the derivative expansion. The GB combination is especially simple to work with in dimensional regularization since there are no propagator corrections in any dimension [15].

For the case of antisymmetric tensors coupled to gravity, another relevant one-loop four-point divergence is that of two gravitons and two antisymmetric tensors, which is generated by the operator

$$
\mathcal{L}_{R H H}=\left(\frac{\kappa}{2}\right)^{2} \frac{1}{(4 \pi)^{2}} \frac{1}{\epsilon} \sqrt{-g} \sum_{j=1}^{n_{2}} R_{\rho \sigma}^{\mu \nu} H_{j \mu \nu \alpha} H_{j}^{\alpha \rho \sigma} .
$$

Like the GB term, this operator is evanescent. In particular, in $D=4$, we can dualize the antisymmetric tensors to scalars, which collapses the Riemann tensor into the Ricci scalar and tensor. Under field redefinitions, they can be eliminated in favor of the dualized scalars, removing the one-loop divergence in two-graviton two-antisymmetrictensor amplitudes with $D=4$ external states. The fourscalar amplitude does diverge.

The change in Eq. (5) under duality transformations is central to the claim by Duff and van Nieuwenhuizen of quantum inequivalence under such transformations [2]. Here, we analyze their effects on the two-loop amplitude. First, let us note that our unitarity-based evaluation of Eq. (5) sews together physical, gauge-invariant tree amplitudes. This explicitly demonstrates that the numerical coefficient of the $R_{\mu \nu \rho \sigma}^{2}$ term in Eq. (5) is gauge invariant, in contrast to implications of Ref. [3]. This gauge invariance suggests that by two loops, Eq. (5) could lead to duality-violating contributions to nonevanescent operators. To see if this happens, we must account for subdivergences and renormalization.

At two loops, pure gravity diverges in $D=4$. The coefficient of this divergence was determined by Goroff and Sagnotti [7] from a three-point computation in the standard $\overline{\mathrm{MS}}$ regularization scheme and later confirmed by van de Ven [8]

$$
\mathcal{L}_{R^{3}}=-\frac{209}{1440}\left(\frac{\kappa}{2}\right)^{2} \frac{1}{(4 \pi)^{4}} \frac{1}{\epsilon} \sqrt{-g} R^{\alpha \beta}{ }_{\gamma \delta} R_{\rho \sigma}^{\gamma \delta} R_{\alpha \beta}^{\rho \sigma},
$$

where we account for the fact that Refs. [7,8] define $\epsilon=4-D$ instead of our $\epsilon=(4-D) / 2$. The divergence in Eq. (7) uses four-dimensional identities to simplify it.

In order to reproduce the Goroff and Sagnotti result, we evaluate the identical-helicity four-graviton amplitude. 
This is the simplest amplitude containing the two-loop divergence (7). While a four-point amplitude may seem to be unnecessarily complicated with respect to a three-point function, there are several advantages to considering an amplitude for a physical process with real momenta. The first is that we can use the unitarity method to obtain a compact integrand [11]. This method is particularly efficient for identical-helicity particles, having been used to obtain compact integrands for the gauge-theory case [13]. More importantly, the question of quantum equivalence under duality transformations can only be properly answered in the context of physical observables, such as renormalized and infrared-subtracted $2 \rightarrow 2$ scattering amplitudes entering physical cross sections.

To facilitate comparisons to the two-loop four-point amplitude, we need the $R^{3}$ divergence (7) inserted into the four-plus-helicity tree amplitude

$$
A_{R^{3}}=\frac{209}{24} \frac{\mathcal{K}}{\epsilon},
$$

where

$$
\mathcal{K} \equiv\left(\frac{\kappa}{2}\right)^{6} \frac{i}{(4 \pi)^{4}} \operatorname{stu}\left(\frac{[12][34]}{\langle 12\rangle\langle 34\rangle}\right)^{2},
$$

and $s=\left(k_{1}+k_{2}\right)^{2}, t=\left(k_{2}+k_{3}\right)^{2}$, and $u=\left(k_{1}+k_{3}\right)^{2}$ are the usual Mandelstam invariants. The last factor is a pure phase constructed from the spinor products $\langle a b\rangle$ and $[a b]$ defined in, for example, Ref. [16].

Figure 1 shows that there are three types of contributions to consider: (a) the bare two-loop contribution, (b) the one-loop single-counterterm subtraction, and (c) the double-counterterm subtraction. One might expect the net subdivergence subtractions (b) and (c) each to be zero because there are no corresponding $D=4$ one-loop divergences. However, this is not correct. A careful analysis of the two-loop integrands [17] reveals subdivergences associated with the GB term (5). For the case of two-forms, a subdivergence corresponding to $\mathcal{L}_{R H H}$ in Eq. (6) must also be subtracted. In principle, when three-forms are present, there might have been subdivergences due to operators containing three-forms, but these do not appear. It is somewhat surprising that there are subdivergences at two loops without any corresponding one-loop divergences in $D=4$. However, because some legs external to the subdivergence are in $D$ dimensions, the cancellations that are specific to $D=4$ do not occur.

While Goroff and Sagnotti also subtracted subdivergences, they did so integral by integral, rather than tracking the operator origin of the subdivergences as we do. Here,

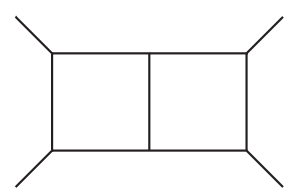

(a)

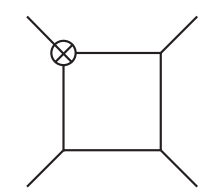

(b)

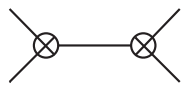

(c)
FIG. 1. Representative diagrams of the (a) bare, (b) singlecounterterm, and (c) double-counterterm insertions. we use dimensional regularization for both infrared and UV divergences; we subtract the well-known infrared singularities [18] from the final result.

We evaluate the bare and single-subtraction contributions via the unitarity method. We take the external legs to be identical-helicity gravitons and each internal leg to be $D$ dimensional. The bare integrand obtained in this way is similar to integrands found earlier for gauge theory $[12,13]$ and for the "double-copy" theory containing a graviton, an antisymmetric tensor, and a dilaton [19]. A key property of these integrands is that they vanish when the loop momenta are taken to reside in $D=4$, yet the amplitudes are still nonvanishing. This phenomenon is related to the observation by Bardeen and Cangemi [20] that the nonvanishing of identical-helicity amplitudes is connected to an anomaly in the self-dual sector.

We follow the same regularization prescriptions used in Ref. [13], where algebraic manipulations on the integrand are performed with $\epsilon<0$. We use the 't Hooft-Veltman variant: We place the external momenta and polarizations in $D=4$ and take the loop momenta and internal states to reside in $D=4-2 \epsilon$ dimensions. Here, we focus on the UV divergences and defer presentation of the integrands and finite terms in the amplitudes to Ref. [17].

We integrate over the loop momenta with the same techniques used to obtain two-loop four-point helicity amplitudes in QCD, including their finite parts [21]. As a cross check, we also directly extract the UV divergences using masses to regulate the infrared [19].

Consider, first, the case of $n_{3}$ three-forms coupled to gravity. In Table I, we give both the divergence and renormalization-scale dependence of each of the three components illustrated in Fig. 1. In the bare and one-loop single-insertion components, the $\ln \mu^{2}$ dependence, where $\mu$ is the renormalization scale, is proportional to the UV divergence. For the bare two-loop part, the $\ln \mu^{2}$ coefficient is twice the coefficient of the $1 / \epsilon$ divergence. For the single counterterm, it is equal to the $1 / \epsilon$ coefficient, and for the double-insertion tree contribution, it vanishes. This follows

TABLE I. Coefficients of the $1 / \epsilon$ UV pole and of $\ln \mu^{2}$ in the identical-helicity four-graviton two-loop amplitude for pure gravity coupled to $n_{3}$ three-forms. We omit the overall factor of $\mathcal{K}$ defined in Eq. (9). The first row gives the bare two-loop contribution, the second row the single GB-counterterm insertion at one loop, and the third row that of a double GB insertion at tree level. The final row gives the total.

\begin{tabular}{lcc}
\hline \hline & $1 / \epsilon$ & $\ln \mu^{2}$ \\
\hline Bare & $-\frac{3431}{5400}-\frac{199}{30} n_{3}+6 n_{3}^{2}$ & $-\frac{3431}{2700}-\frac{199}{15} n_{3}+12 n_{3}^{2}$ \\
GB & $\frac{4 \cdot 53-180 n_{3} \frac{2 \cdot\left(13+180 n_{3}\right)}{360}}{15}$ & $\frac{689}{675}+\frac{199}{15} n_{3}-12 n_{3}^{2}$ \\
Double GB & $24\left(\frac{4 \cdot 53-180 n_{3}}{360}\right)^{2}$ & 0 \\
Total & $\frac{209}{24}-\frac{15}{2} n_{3}$ & $-\frac{1}{4}$ \\
\hline \hline
\end{tabular}


TABLE II. Coefficients of the $1 / \epsilon$ UV pole and of $\ln \mu^{2}$ in the four-graviton amplitude for gravity coupled to $n_{0}$ scalars. The table follows the same format as Table I.

\begin{tabular}{lcc}
\hline \hline Bare & $1 / \epsilon$ & $\ln \mu^{2}$ \\
GB & $-\frac{3431}{5400}-\frac{277}{10800} n_{0}+\frac{n_{0}^{2}}{5400}$ & $-\frac{3431}{2700}-\frac{277}{5400} n_{0}+\frac{n_{0}^{2}}{2700}$ \\
Double GB & $\frac{4 \cdot 53+n_{0}}{360} \frac{2 \cdot\left(13-n_{0}\right)}{15}$ & $\frac{689}{675}-\frac{199}{2700} n_{0}-\frac{n_{0}^{2}}{2700}$ \\
Total & $24\left(\frac{4 \cdot 53+n_{0}}{360}\right)^{2}$ & 0 \\
\hline \hline
\end{tabular}

from dimensional analysis of the loop integrals, with measure $\int d^{4-2 \epsilon} \ell$ per loop, requiring an overall factor of $\mu^{2 L \epsilon}$ at $L$ loops. The counterterm subtractions are pure poles that do not carry such factors. In the sum over terms, there is no simple relation between the $1 / \epsilon$ and the $\ln \mu^{2}$ coefficients, in contrast to many textbook examples at one loop.

As seen from the last line of Table I, with no threeform fields, we match exactly the Goroff and Sagnotti divergence (8). The addition of $n_{3}$ three-form fields shifts the divergence from the pure gravity result. One might think that this shift would lead to a physical change in the scattering amplitudes through a different dependence on $\mu$. However, the $\ln \mu^{2}$ column of Table I shows that the $n_{3}$ dependence of the bare and single-counterterm contributions precisely cancels in the sum. The scale dependence is, therefore, unaffected by three-form fields. The differences in the divergent parts can be removed by adjusting the coefficient of the $(1 / \epsilon) R^{3}$ counterterm. We have also obtained the amplitude's finite parts [17]. Their form allows for a finite $R^{3}$ subtraction that completely eliminates the effects of three-form fields in the two-loop renormalized identical-helicity amplitude.

We now turn to the case of duality transformations between antisymmetric-tensor fields and scalars. In Tables II and III, the coefficients of $1 / \epsilon$ and $\ln \mu^{2}$ terms are collected. The tables show that, while the individual components are quite different and the final $1 / \epsilon$ divergence changes under duality transformations, scalars and twoforms have exactly the same renormalization-scale dependence. As for the case of three-forms, we find that the UV divergence does depend on the field representations, but the renormalization-scale dependence does not. Again, finite subtractions can be found to make the dual pair of renormalized amplitudes identical [17].

From Tables I-III, we find that, in all cases, the scale dependence in the identical-helicity four-graviton amplitude follows a simple behavior,

$$
\left.\mathcal{M}_{4}^{(2)}\right|_{\ln \mu^{2}}=-\mathcal{K} \frac{N_{b}-N_{f}}{8} \ln \mu^{2},
$$

where $N_{b}\left(N_{f}\right)$ is the number of bosonic (fermionic) fourdimensional states in the theory. We only computed Eq. (10) explicitly for $N_{f}=0$, but the identical-helicity
TABLE III. Coefficients of the $1 / \epsilon \mathrm{UV}$ pole and of $\ln \mu^{2}$ in the two-loop four-graviton amplitude for gravity coupled to $n_{2}$ antisymmetric-tensor fields. The table follows the same format as Table I. The second-to-last row gives the contribution of the RHH counterterm inserted into the one-loop amplitude.

\begin{tabular}{lcc}
\hline \hline & $1 / \epsilon$ & $\ln \mu^{2}$ \\
\hline Bare & $-\frac{3431}{5400}+\frac{8543}{10800} n_{2}+\frac{8281}{5400} n_{2}^{2}$ & $-\frac{3431}{2700}+\frac{8543}{5400} n_{2}+\frac{8281}{2700} n_{2}^{2}$ \\
GB & $\frac{4 \cdot 53+91 n_{2} 2 \cdot\left(13-91 n_{2}\right)}{360}$ & $\frac{689}{675}-\frac{18109}{2700} n_{2}-\frac{8281}{2700} n_{2}^{2}$ \\
Double & $24\left(\frac{4 \cdot 53+91 n_{2}}{360}\right)^{2}$ & 0 \\
GB & $\frac{209}{5 n_{2}}+\frac{299}{48} n_{2}$ & $5 n_{2}$ \\
RHH & Total & $-\frac{2+n_{2}}{8}$ \\
\hline \hline
\end{tabular}

graviton amplitude vanishes in supersymmetric theories, forcing Eq. (10) to be proportional to $N_{b}-N_{f}$.

The $\ln \mu^{2}$ dependence is clearly a more appropriate quantity for deciding whether a theory should be thought of as nonrenormalizable. If the coefficient of the $\ln \mu^{2}$ is nonvanishing, as is the case for pure gravity, the coefficient will run, and we consider such a theory to be nonrenormalizable. Our result shows that, instead of focusing on the divergences, one should study the $\ln \mu^{2}$ coefficient to see if there is a principle that can be applied to set it to zero. One obvious useful principle is that renormalization schemes should be chosen that maintain the equality of theories related by duality transformations.

In this light, one might wonder if the recently computed four-loop divergence of pure $\mathcal{N}=4$ supergravity [22] is an artifact of the particular SU(4) formulation of the theory that was used. However, with the uniform mass infrared regulator used in that calculation, extensive checks reveal that all subdivergences cancel. Therefore, the coefficient of $\ln \mu^{2}$ is proportional to that of the $1 / \epsilon$ divergence. When matter multiplets are added there are one-loop subdivergences, but those are not evanescent. In other formulations, it is possible that the divergences will change, but we do not expect the $\ln \mu^{2}$ coefficients to change.

In summary, our investigation of the ultraviolet divergences of nonsupersymmetric gravity reveals a number of striking features. The first is the nontrivial role of the trace anomaly and the associated evanescent Gauss-Bonnet term entering subdivergences. It is remarkable that a term that vanishes in four dimensions can contribute directly to the leading divergence of a graviton amplitude. Another important feature is that the integrand of the identicalhelicity amplitude vanishes if the loop momenta are taken to be four dimensional; this feature of identical-helicity amplitudes, which follows straightforwardly from unitarity, is also tied to anomalous behavior [20]. Similar connections to anomalous behavior [23] were noted in the fourloop divergence of $\mathcal{N}=4$ pure supergravity [22].

A key lesson is that, under duality transformations, the values of two-loop divergences can change, contrary to the 
situation at one loop [5]. However, the difference in these divergences is unphysical, in the sense that it can be absorbed into a redefinition of the coefficient of a local operator. In other words, our results for scattering amplitudes are consistent with quantum equivalence under duality transformations when that equivalence allows for the adjustment of coefficients of higher-dimension operators. The dependence on the renormalization scale does not change under duality transformations in the examples we studied; it is a more appropriate measure of the UV properties of the theory. It would be quite interesting to establish this property beyond two loops. Together with recent examples of ultraviolet finiteness in supergravity amplitudes, despite the existence of seemingly valid counterterms [24,25], the results summarized in this Letter show that much more remains to be learned about both duality at the quantum level and the ultraviolet structure of gravity theories.

We especially thank David Kosower, Radu Roiban, Augusto Sagnotti, and Raman Sundrum for many useful and interesting discussions and suggestions. We also thank Luis Alvarez-Gaume, John Joseph Carrasco, Stanley Deser, Paolo Di Vecchia, Gary Horowitz, Henrik Johansson, Tim Jones, Kelly Stelle, and Mark Wise for helpful discussions. This material is based upon work supported by the Department of Energy under Award No. DE-SC0009937 and Contract No. DE-AC02-76SF00515 and the Gordon and Betty Moore Foundation through Grant No. 776 to the Caltech Moore Center for Theoretical Cosmology and Physics. C. C. is supported by DOE Early Career Award No. DE-SC0010255 and a Sloan Research Fellowship. Z. B. and L. D. are grateful to the Simons Foundation for support and to the Walter Burke Institute at Caltech for hospitality. C. C. and L. D. thank the Aspen Center for Physics for hospitality and the NSF for support through Grant No. 1066293. S. D. and J. N. gratefully acknowledge Mani Bhaumik for his generous support.

[1] D. M. Capper and M. J. Duff, Trace anomalies in dimensional regularization, Nuovo Cimento A 23, 173 (1974); Conformal anomalies and the renormalizability problem in quantum gravity, Phys. Lett. A 53, 361 (1975); H.S. Tsao, Conformal anomalies in a general background metric, Phys. Lett. B 68, 79 (1977); G. W. Gibbons, S. W. Hawking, and M. J. Perry, Path integrals and the indefiniteness of the gravitational action, Nucl. Phys. B138, 141 (1978); R. Critchley, Trace anomaly for gravitons, Phys. Rev. D 18, 1849 (1978); M. J. Duff, Twenty years of the Weyl anomaly, Classical Quantum Gravity 11, 1387 (1994).

[2] M. J. Duff and P. van Nieuwenhuizen, Quantum inequivalence of different field representations, Phys. Lett. B 94, 179 (1980).

[3] W. Siegel, Quantum equivalence of different field representations, Phys. Lett. B 103, 107 (1981).

[4] E. S. Fradkin and A. A. Tseytlin, Quantum equivalence of dual field theories, Ann. Phys. (N.Y.) 162, 31 (1985); M. T. Grisaru, N. K. Nielsen, W. Siegel, and D. Zanon, Energy momentum tensors, supercurrents, (super)traces and quantum equivalence, Nucl. Phys. B247, 157 (1984).

[5] E. Sezgin and P. van Nieuwenhuizen, Renormalizability properties of antisymmetric tensor fields coupled to gravity, Phys. Rev. D 22, 301 (1980); E. S. Fradkin and A. A. Tseytlin, One loop infinities in dimensionally reduced supergravities, Phys. Lett. B 137, 357 (1984).

[6] G. 't Hooft and M. J. G. Veltman, One loop divergencies in the theory of gravitation, Ann. Inst. Henri Poincare, Sect. A 20, 69 (1974).

[7] M. H. Goroff and A. Sagnotti, Quantum gravity at two loops, Phys. Lett. B 160, 81 (1985); The ultraviolet behavior of Einstein gravity, Nucl. Phys. B266, 709 (1986).

[8] A. E. M. van de Ven, Two loop quantum gravity, Nucl. Phys. B378, 309 (1992).

[9] A. J. Buras and P. H. Weisz, QCD nonleading corrections to weak decays in dimensional regularization and 't HooftVeltman schemes, Nucl. Phys. B333, 66 (1990); M. J. Dugan and B. Grinstein, On the vanishing of evanescent operators, Phys. Lett. B 256, 239 (1991); I. Jack, D. R. T. Jones, and K.L. Roberts, Equivalence of dimensional reduction and dimensional regularization, Z. Phys. C 63, 151 (1994); S. Herrlich and U. Nierste, Evanescent operators, scheme dependences and double insertions, Nucl. Phys. B455, 39 (1995); R. Harlander, P. Kant, L. Mihaila, and M. Steinhauser, Dimensional reduction applied to QCD at three loops, J. High Energy Phys. 09 (2006) 053.

[10] E. S. Fradkin and A. A. Tseytlin, Renormalizable asymptotically free quantum theory of gravity, Phys. Lett. B 104, 377 (1981); Renormalizable asymptotically free quantum theory of gravity, Nucl. Phys. B201, 469 (1982); M. B. Einhorn and D. R. T. Jones, Gauss-Bonnet coupling constant in classically scale-invariant gravity, Phys. Rev. D 91, 084039 (2015)

[11] Z. Bern, L. J. Dixon, D. C. Dunbar, and D. A. Kosower, One loop $\mathrm{n}$ point gauge theory amplitudes, unitarity and collinear limits, Nucl. Phys. B425, 217 (1994); Fusing gauge theory tree amplitudes into loop amplitudes, Nucl. Phys. B435, 59 (1995); Z. Bern, L. J. Dixon, D. C. Dunbar, M. Perelstein, and J.S. Rozowsky, On the relationship between YangMills theory and gravity and its implication for ultraviolet divergences, Nucl. Phys. B530, 401 (1998).

[12] Z. Bern and A. G. Morgan, Massive loop amplitudes from unitarity, Nucl. Phys. B467, 479 (1996); Z. Bern, L. J. Dixon, and D. A. Kosower, Progress in one loop QCD computations, Annu. Rev. Nucl. Part. Sci. 46, 109 (1996).

[13] Z. Bern, L. J. Dixon, and D. A. Kosower, A two loop four gluon helicity amplitude in QCD, J. High Energy Phys. 01 (2000) 027.

[14] J. D. Brown and C. Teitelboim, Dynamical neutralization of the cosmological constant, Phys. Lett. B 195, 177 (1987); Neutralization of the cosmological constant by membrane creation, Nucl. Phys. B297, 787 (1988); R. Bousso and J. Polchinski, Quantization of four-form fluxes and dynamical neutralization of the cosmological constant, J. High Energy Phys. 06 (2000) 006.

[15] B. Zwiebach, Curvature squared terms and string theories, Phys. Lett. B 156, 315 (1985).

[16] M. L. Mangano and S. J. Parke, Multiparton amplitudes in gauge theories, Phys. Rep. 200, 301 (1991).

[17] Z. Bern, C. Cheung, H.-H. Chi, S. Davies, L. Dixon, and J. Nohle (to be published). 
[18] S. Weinberg, Infrared photons and gravitons, Phys. Rev. 140, B516 (1965); S. G. Naculich and H. J. Schnitzer, Eikonal methods applied to gravitational scattering amplitudes, J. High Energy Phys. 05 (2011) 087; S. G. Naculich, H. Nastase, and H. J. Schnitzer, All-loop infrared-divergent behavior of most-subleading-color gauge-theory amplitudes, J. High Energy Phys. 04 (2013) 114; R. Akhoury, R. Saotome, and G. Sterman, Collinear and soft divergences in perturbative quantum gravity, Phys. Rev. D 84, 104040 (2011).

[19] Z. Bern, S. Davies, T. Dennen, Y.-t. Huang, and J. Nohle, Color-kinematics duality for pure Yang-Mills and gravity at one and two loops, Phys. Rev. D 92, 045041 (2015).

[20] W. A. Bardeen, Selfdual Yang-Mills theory, integrability and multiparton amplitudes, Prog. Theor. Phys. Suppl. 123, 1 (1996); D. Cangemi, Selfdual Yang-Mills theory and one loop like-helicity QCD multi-gluon amplitudes, Nucl. Phys. B484, 521 (1997); Selfduality and maximally helicity violating QCD amplitudes, Int. J. Mod. Phys. A 12, 1215 (1997).

[21] V. A. Smirnov and O. L. Veretin, Analytical results for dimensionally regularized massless on-shell double boxes with arbitrary indices and numerators, Nucl. Phys. B566, 469 (2000); C. Anastasiou, T. Gehrmann, C. Oleari,
E. Remiddi, and J. B. Tausk, The tensor reduction and master integrals of the two-loop massless crossed box with light-like legs, Nucl. Phys. B580, 577 (2000); Z. Bern, A. De Freitas, and L. J. Dixon, Two loop helicity amplitudes for gluon-gluon scattering in QCD and supersymmetric Yang-Mills theory, J. High Energy Phys. 03 (2002) 018.

[22] Z. Bern, S. Davies, T. Dennen, A. V. Smirnov, and V. A. Smirnov, Ultraviolet Properties of $\mathcal{N}=4$ Supergravity at Four Loops, Phys. Rev. Lett. 111, 231302 (2013).

[23] N. Marcus, Composite anomalies in supergravity, Phys. Lett. B 157, 383 (1985); J. J. M. Carrasco, R. Kallosh, R. Roiban, and A. A. Tseytlin, On the U(1) duality anomaly and the S-matrix of $(\mathcal{N})=4$ supergravity, J. High Energy Phys. 07 (2013) 029.

[24] Z. Bern, S. Davies, T. Dennen, and Y.-t. Huang, Absence of Three-Loop Four-Point Divergences in $\mathcal{N}=4$ Supergravity, Phys. Rev. Lett. 108, 201301 (2012); Ultraviolet cancellations in half-maximal supergravity as a consequence of the double-copy structure, Phys. Rev. D 86, 105014 (2012).

[25] Z. Bern, S. Davies, and T. Dennen, Enhanced ultraviolet cancellations in $\mathcal{N}=5$ supergravity at four loops, Phys. Rev. D 90, 105011 (2014). 